At baseline, BMI was inversely associated with physical and mental SF-12 summary scores $(\beta[95 \% \mathrm{CI}]=-0.3[-0.4$ to -0.2$]$, and -0.1 [-0.1 to 0.0]). An increase in BMI of $1 \mathrm{~kg} / \mathrm{m}^{2}$ within 12 months was associated with a significant decrease in physical but not mental SF12 scores $(0.3[-0.4$ to -0.1$])$. Due to an interaction with gender, the association between change in BMI and physical SF-12 scores was only prevalent in women $(\beta=-0.5[-0.7$ to -0.2$], p<0.001)$ but not in men $(\mathrm{p}=0.32)$.

Conclusion BMI is inversely associated with quality of life in highrisk cardiovascular patients. Increases in BMI may lead to a decrease in physical quality of life in women but not in men.

\section{P2-205 HETEROGENEITY IN FUNCTIONAL RECOVERY AFTER STROKE: AN EXPLORATORY STUDY USING LONGITUDINAL LATENT CLASS ANALYSIS}

doi:10.1136/jech.2011.142976j.38

T Munyombwe, ${ }^{*}$ R West, K Hill, Y-K Tu, P Knapp. University of Leeds, Leeds, UK

Introduction Prediction models for functional recovery after stroke can be improved by adjusting for the heterogeneity in functional recovery patterns. This study explored the heterogeneity in functional recovery after stroke using longitudinal latent class analysis and characterised the patients in the different latent classes.

Methods The analyses were performed on a data set from a cohort of 448 stroke survivors participating in a study of outcomes at 1 year. Heterogeneity in functional recovery after stroke was investigated using Longitudinal Latent class analysis of total Barthel scores measured at 1, 6 and 12 months after stroke. Identification of the optimal number of classes was based on BIC, AIC, and LoMendell-Rubin Adjusted Likelihood ratio test. The second analysis characterised the latent classes.

Results A four latent class structure was preferred. All the four latent classes showed a non linear pattern of recovery over time. Persons in the very poor functional recovery group had the largest median length of initial hospital stay 99 (13-257 days), mean age $75 \pm 9.27$ years and greatest probability of being urinary and bladder incontinence. The group with best functional recovery had the least initial hospital stay 14 (2-147 days), least proportion of people with previous stroke, least proportion of people with urinary and bladder incontinence, the mean age at admission was $68.76 \pm 11.72$ years.

Conclusion The study showed that there is heterogeneity in functional recovery patterns after stroke. Latent class analysis is a useful method for identifying subgroups of functional recovery after stroke.

\section{P2-206 ASSOCIATION OF FAAR SCORE ON ADMISSION ECG WITH MORTALITY IN 1843 PATIENTS ADMITTED WITH AN ACUTE CORONARY SYNDROME}

doi:10.1136/jech.2011.142976j.39

\begin{abstract}
${ }^{1} \mathrm{M}$ Lown, ${ }^{2} \mathrm{~T}$ Munyombwe, ${ }^{*} \mathrm{~W}$ Harrison, ${ }^{2} \mathrm{R}$ West, ${ }^{1} \mathrm{C}$ Hall, ${ }^{1} \mathrm{C}$ Morrell, ${ }^{1} \mathrm{~B}$ Jackson, ${ }^{1} \mathrm{R}$ Sapsford, ${ }^{1} \mathrm{~N}$ Kilcullen, ${ }^{1} \mathrm{C}$ Pepper, ${ }^{3} \mathrm{P}$ Batin, ${ }^{1} \mathrm{~A}$ Hall, ${ }^{1,2} \mathrm{C}$ Gale, ${ }^{5} \mathrm{~A}$ Simms. ${ }^{1}$ University of Leeds, Center for Epidemiology and Biostatistics, Leeds, UK; ${ }^{2}$ Department of Cardiology, Leeds Teaching Hospitals NHS Trust, Leeds, UK; ${ }^{3}$ Department of Cardiology, Mid-Yorkshire NHS Trust, Wakefield, Wakefield, UK; ${ }^{4}$ Department of Cardiology, York Hospitals NHS Foundation Trust, York, UK; ${ }^{5}$ Leeds General Infirmary, Leeds, UK
\end{abstract}

Introduction Accurate risk assessment is central to the management of patients presenting with acute coronary syndromes (ACS). Many ACS risk scores are employed as near-point tests, but rely on the collection of remote data such as troponin concentration. This study investigated the long term and short term mortality prediction performance of the Frontal ORS-T Angle and Age Risk (FAAR) score, a simple ACS risk stratification tool comprising of the frontal ORS$T$ angle and age at admission and compared with GRACE risk score which relies on a multitude of clinical variables.

Method Data from the Evaluation of Methods and Management of Acute Coronary Events EMMACE-2 and EMMACE-1 prospective studies was used to test and validate models respectively. EMMACE studies examined outcomes in consecutively admitted, unselected patients with confirmed ACS in multiple adjacent hospitals (within the catchment area of one tertiary centre) in Yorkshire, UK. Using the EMMACE-2 (2499 patients), FAAR score adjusted for patient characteristics, a stepwise logistic regression and proportional hazards Cox regression models was used to predict 30-day and 2 year mortality.

Results The FAAR score offered excellent discriminative performance for 30-day, C statistic (95\% CI) 0.74 (0.71 to 0.78) and 2-year 0.76 (0.74 to 0.78 ) mortality, maintained its performance in the EMMACE-1 validation cohort at 30-days, C statistic (95\% CI) 0.76 (0.71 to 0.80$)$ and at 2 -years 0.79 (0.75 to 0.83 ), and compared favourably with the GRACE score.

Conclusion FAAR score could be used by emergency healthcare professionals to assist the triage of patients presenting with suspected ACS.

\section{P2-207 EPIDEMIOLOGY OF NON-COMMUNICABLE DISEASES AND THEIR RISK FACTORS IN RURAL UGANDA}

doi:10.1136/jech.2011.142976j.40

${ }^{1} \mathrm{G}$ Murphy, ${ }^{* 2} \mathrm{R}$ Nsubuga, ${ }^{2} \mathrm{G}$ Asiki, ${ }^{2,4} \mathrm{~J}$ Seeley, ${ }^{1,3} \mathrm{E}$ Young, ${ }^{2,5} \mathrm{~A}$ Kamali. ${ }^{1}$ University of Cambridge, Cambridge, UK; ${ }^{2}$ MRC/UVRI Uganda Research Unit on AIDS, Entebbe, Uganda; ${ }^{3}$ Wellcome Trust Sanger Institute, Hinxton, UK; ${ }^{4}$ University of East Anglia, Norwich, UK; ${ }^{5}$ London School of Hygiene and Tropical Medicine, London, UK

Introduction Non-communicable diseases (NCDs) are rapidly becoming leading causes of morbidity and death in low- and middleincome countries, including those in sub-Saharan Africa. Yet, the magnitude and distribution of established and emerging risk factors for NCDs have not been fully studied in sub-Saharan African countries in a large scale epidemiological context.

Methods We will carry out a cross-sectional population-based survey of cardiometabolic risk factors and disease of approximately 8000 participants ages 13 years and older, in a rural population in Uganda. Trained field staff will conduct a questionnaire based upon the WHO STEPwise approach to Surveillance questionnaire; perform biophysical measurement, including anthropometry and blood pressure readings; and collect blood samples for biochemical analysis for cardiometabolic risk factors and infection.

Results By August 2011, data on approximately 6000 participants will have been collected. We will report initial findings, including the prevalence and distribution of lifestyle risk factors, physical measurements and biochemical measurements. Lifestyle risk factors will include tobacco use, alcohol consumption, diet and physical activity. Physical measurements will include blood pressure, body mass index and waist hip ratio, as indicators of hypertension and obesity, respectively. Biochemical measurements will include liver function tests, lipid levels and $\mathrm{HbA1c}$ as an indication of diabetes. Age and sex specific estimates will be reported.

Conclusion Population based epidemiological studies can provide reliable data on disease burden and their risk factors to help inform public health policy and programmes aimed at addressing the rise in NCDs in Uganda. 